\title{
Percutaneous Tracheostomy Coming of Age for the Neurointensivist?
}

\author{
Dale Hoekema
}

Published online: 20 February 2009

(C) Humana Press Inc. 2009

In June 2009 it will be 24 years since the landmark article by New York's Dr. Ciaglia was published in Chest regarding: "A New Simple Bedside Procedure; Elective Percutaneous Dilatation Tracheostomy" [1]. Since its introduction PT (Percutaneous Tracheostomy) has made an significant impact on the way we manage critical care patients particularly in today's Neurocritical Care Units.

In this issue of the journal Sedar et al. also from New York report a retrospective cohort review of ST (open surgical tracheostomy) performed by surgeons compared to PT performed by Neurointensivists. The patient population at Columbia University Medical Center in New York represented a typical Neurocritical Care Unit group of diagnoses although there were no patients with spinal cord injury and relatively fewer with traumatic brain injury than other NCCU (Neurocritical Care Units). In the past 24 years dozens of papers and thousands of patients have been studied both retrospectively and prospectively with regard to the question of ST versus PT with many studies looking at intensivists rather than surgeons being the operators performing the PT. Sedar et al., however, are the first to publish a large series where the PT was performed by Neurointensivists.

Benefits of the PT over the ST are numerous and include: no need to move the patient to the OR (operating room), and the procedure historically can be performed sooner resulting in decreased NCCU length of stay with a reduction in cost of approximately $\$ 33,000$ per patient in Seder's review (an average of four ICU days less for the PT patients) $P$ value (0.01). Browd et al. from the University

D. Hoekema $(\bowtie)$

Neurocritical Care Unit, University of Mississippi Medical Center, Jackson, USA

e-mail: dhoekema@neurosurgery.umsmed.edu of Utah reported in this journal in 2005 a series of 43 patients with PT performed by the Neurosurgical faculty demonstrating approximately $\$ 1000.00$ cost savings for the procedure itself [2]. In Seder's series there was a trend towards a lower respiratory infection rate but it did not reach statistical significance $P$ value $(0.1)$.

However, it is intuitive that oral secretions will track down the endotracheal tube resulting in a higher rate of ventilator associated pneumonia than in patients with a tracheostomy who are able to swallow their secretions. The scar is usually smaller in patients with a PT with a more acceptable cosmetic result. Yilmaz et al. [3] reported in this journal in 2006 a series of 12 patients where a repeated PT was performed without complications.

The acceptance and feasibility of PT performed by intensivists is international with articles from Australia [4], England [5], Israel [6], Canada [7], and the Netherlands [8] to mention a few. In 2006 in Australia Silvester et al. [4] published the largest prospective randomized controlled trial to date comparing 100 patients with PT versus ST. In their series the PT was performed by intensivists and the ST was performed at the bedside by surgeons. Complications were minimal with both types of procedures and included bleeding 3-4\%, pneumothorax $<1 \%$, accidental decanulation $<1 \%$, tracheostomy site infection $1-3 \%$, posterior wall of trachea injury $0 \%$, and tracheal stenosis $0 \%$. There were no cases of a false passage creation and there were no cases of loss of the air way with or without death. Although long term follow-up was done at 20 months with flow volume loops and bronchoscopy it included only 29 patients in the PT group and 42 patients in the ST group. Approximately $30-35 \%$ of patients died prior to discharge from the hospital with approximately $10 \%$ being discharged with the tracheostomy. The mean duration of the tracheostomy prior to decanulation was 
about 3 weeks for both groups. Silvester et al. concluded that PT and ST both had similar and low complication rates. PT was performed more quickly and had a better cosmetic result. They underscored the importance of the training and skill of the operator for either procedure. Seder et al. reported a similar complication rate as Silvester but without long term follow-up.

Local politics dictate in many institutions who performs the tracheotomies on the critical care patients. However, it is very clear from the publications and experience of intensivists including Neurologists, Anesthesiologists, and Pulmonary Critical Care physicians that they can be trained in the procedure and attain excellence in its performance. Now is the time as Neurocritical Care is coming of age that Neurocritical Care physicians acquire the training and claim their right to take care of their own patients including percutaneous tracheostomy.

Challenges for Neurointensivists in the future who plan to train fellows to perform PT include achieving the expertise to demonstrate the low complication rates and safety noted in the Silvester and Sedar series listed above. The use of the bronchoscope is felt by some to be mandatory but many including myself, Sedar, and some of the Intensivists in the Silvester series prefer to perform the procedure with the bronchoscope on standby only to be used if needed. We have developed a technique using a bronchoscopy adapter on the endotracheal tube through which we place a $5 \mathrm{~mm}$ Cook tube changer which extends $10 \mathrm{~cm}$ beyond the tip of the endotracheal tube allowing easy rescue of the air way if needed. The tube changer is small enough not to impair ventilation (important for ICP control) during the procedure or interfere with accessing the trachea and placing the tracheostomy. PT is now really a misnomer since most operators use a modified technique creating a $1.5-2 \mathrm{~cm}$ horizontal incision a fingers breadth below the cricoid cartilage. Blunt dissection is carried out to the anterior tracheal wall with a hemostat and gloved finger. Our preference is to leave the cuff on the endotracheal tube inflated as it is drawn back $8 \mathrm{~cm}$ from its original position.

We place the needle for canulation on the trachea while it is splinted by the cuff of the endotracheal tube. When the cuff passes under the gloved finger held on the trachea beside the needle, the needle is inserted and air is aspirated and the guide wire is passed, etc.

Despite Silvester's and Sedar's results showing no complication of tracheal stenosis, our experience and the literature confirm there is a definite prevalence of tracheal stenosis following PT requiring interventions from our ENT (Ear, Nose, and Throat) colleagues. It still remains controversial if this complication is more common following PT than ST [9, 10]. Tracheal stenosis may be under reported since many of our patients after leaving the
Neurocritical Care Unit are not seen again by the Intensivists and are lost to our follow-up. The Cook company after marketing the "Blue Rhino" ostensibly because of concern about long term complications of tracheal stenosis has more recently marketed the "Blue Dolphin" which is supposed to reduce the risk of late tracheal stenosis by minimizing the trauma to the tracheal wall. It creates the stoma in the tracheal wall with balloon dilatation. Some ENT colleagues have advocated the development of a device to use with the PT to remove a button of tissue from the anterior tracheal wall to avoid it being displaced posteriorly into the tracheal lumen setting up the risk for tracheal stenosis in PT. To my knowledge no one has yet developed such a device.

Over the last 8 years in the 16 bed Neurocritical Care Unit at the University of Mississippi I have personally performed over 300 PT's. My complications have been minimal with no significant bleeding or infections or other problems, except one patient who developed tracheal stenosis as a late complication and required reconstructive tracheal surgery. We have recently developed a registry for these patients to track complications as well as the natural history of the PT. We encourage others to consider the same in order that Neurointensivists may have a more accurate and accountable record regarding complications. We also suggest that the Neurocritical Care Society develop a monograph and a course on the subject of PT to maintain a standard of excellence in teaching Neurocritical Care fellows and other Neurointensivists this important procedure.

\section{References}

1. Ciaglia P, Firsching R, Syniec C. Elective percutaneous dilatational tracheostomy. A new simple bedside procedure; preliminary report. Am Coll Chest Physicians. 1985;87:715-9.

2. Browd SR, MacDonald JD. Percutaneous dilatational tracheostomy in neurosurgical patients. Neurocrit Care. 2005;2:268-73. doi:10.1385/NCC:2:3:268.

3. Yilmaz M, Dosemeci L, Cengiz M, Sanli S, et al. Repeat percutaneous tracheostomy in the neurocritically ill patient. Neurocrit Care. 2006;05:120-3. doi:10.1385/NCC:5:2:120.

4. Silvester W, Goldsmith D, Uchino S, Bellomo R, et al. Percutaneous versus surgical tracheostomy: a randomized controlled study with long-term follow-up. Crit Care Med. 2006;34(8): 2145-52. doi:10.1097/01.CCM.0000229882.09677.FD.

5. Patel A, Swan P, Dunning J. Does percutaneous tracheostomy have a lower incidence of complications compared to an open surgical technique? Interact Cardiovasc Thorac Surg. 2005;4: 563-8. doi:10.1510/icvts.2005.117697.

6. Nun AB, Orlovsky M, Best LA. Percutaneous tracheostomy in patients with cervical spine fractures-feasible and safe. Interact Cardiovasc Thorac Surg. 2006;5:427-9. doi:10.1510/icvts.2005. 125690.

7. Higgins KM, Punthakee X. Meta-analysis comparison of open versus percutaneous tracheostomy, The American Laryngological. Rhinol Otol Soc. 2007;117:447-54. 
8. Polderman KH, Spijkstra JJ, de Bree R, et al. Percutaneous dilatational tracheostomy in the ICU. Chest. 2003;123:1595-602. doi:10.1378/chest.123.5.1595.

9. Massick DD, Yao S, Powell DM, et al. Bedside Tracheostomy in the Intensive Care Unit: a prospective randomized trial comparing open surgical tracheostomy with endoscopically guided percutaneous dilatational tracheostomy, The American Laryngological. Rhinol Otol Soc. 2001;111:494-500.

10. Freeman BD, Isabella $\mathrm{K}$, Cobb $\mathrm{P}$, et al. A prospective, randomized study comparing percutaneous with surgical tracheostomy in critically ill patients. Crit Care Med. 2001;29:926-30. doi:10.1097/ 00003246-200105000-00002. 